\title{
Study of invisible neutrino decay and oscillation in the presence of matter with a 50 kton magnetised iron detector
}

\author{
S. Choubey ${ }^{a, b, c}$, S. Goswami ${ }^{d}$, C. Gupta ${ }^{b, d}$, Lakshmi.S.Mohan $^{* d} \dagger$, T. Thakore ${ }^{e}$ \\ ${ }^{a}$ Harish-Chandra Research Institute, Chhatnag Road, Jhunsi, Allahabad 211 019, India \\ ${ }^{b}$ KTH Royal Institute of Technology, AlbaNova University Center, 10691 Stockholm, Sweden, \\ ${ }^{c}$ Homi Bhabha National Institute, Mumbai 400085, India, \\ ${ }^{d}$ Physical Research Laboratory, Navrangpura, Ahmedabad 380 009, India \\ ${ }^{e}$ Louisiana State University, Baton Rouge 70803, LA \\ E-mail: sandhya@hri.res.in, sruba@prl.res.in, chandang@prl.res.in, \\ ph17ipf06@smail.iitm.ac.in, tarakstar@gmail.com
}

\begin{abstract}
The sensitivity to the invisible decay of the mass eigenstate $\nu_{3}$ in the presence of Earth matter effects is studied. Only the charged current interactions of atmospheric $\nu_{\mu}$ and $\bar{\nu}_{\mu}$ for $500 \mathrm{kTon}$ year exposure of a future magnetised iron detector at INO are analysed. The analysis with observed muon energy in the range would give a constraint of $\tau_{3} / m_{3}>1.51 \times 10^{-10} \mathrm{~s} / \mathrm{eV}$ at $90 \% \mathrm{CL}$ with this exposure. Here $\tau_{3}$ is the lifetime and $m_{3}$ is the mass of $\nu_{3}$, when it is the heaviest. The effect of decay on the precision measurement of $\sin ^{2} \theta_{23}$ and $\left|\Delta m_{32}^{2}\right|$ and neutrino mass hierarchy are also studied. Since the presence of decay affects the oscillation amplitude rather than its phase, it is seen that the precision on $\sin ^{2} \theta_{23}$ worsens whereas that on $\left|\Delta m_{32}^{2}\right|$ is not much affected. Sensitivity to hierarchy also worsens slightly in the presence of the invisible decay of $\nu_{3}$.
\end{abstract}

The 19th International Workshop on Neutrinos from Accelerators-NUFACT2017

25-30 September, 2017

Uppsala University, Uppsala, Sweden

\footnotetext{
${ }^{*}$ Speaker.

${ }^{\dagger}$ Currently at Indian Institute of Technology Madras, Chennai
} 


\section{Introduction}

Flavour oscillations of neutrinos have been established from the observation of neutrino oscillations at various energies and baselines by different experiments over the world. Currently, the major open questions in neutrino oscillation physics are neutrino mass hierarchy, octant of the mixing angle $\theta_{23}$ and the value of the CP phase $\delta_{C P}$. The Iron Calorimeter (ICAL) detector $[1,2]$ at the proposed India-based Neutrino Observatory (INO) is an atmospheric neutrino experiment aiming to study the 2-3 oscillation parameters by probing the Earth matter effects on the propagation of atmospheric neutrinos. The main goal of ICAL is to determine neutrino mass hierarchy and to perform precision measurements on $\sin ^{2} \theta_{23}$ and $\left|\Delta m^{2}{ }_{32}\right|$. New physics phenomena are expected to change the oscillation probabilities and will affect the number of observed events. Hence oscillation experiments can be used to study the sensitivity to the new physics parameter itself. The presence of the new physics parameter will also affect the determination of the standard oscillation parameters and thus creating new degeneracies. Here we investigate one such phenomenon namely the invisible decay of the mass eigen state $\nu_{3}$ while its travel from its production point to the detector. For this simulation study we have used charged current $\nu_{\mu}$ and $\bar{\nu}_{\mu}$ events obtained with $500 \mathrm{kTon}$ year exposure of INO ICAL to atmospheric neutrinos whose oscillation probabilities in matter are modified by the invisible decay model we consider. Here the mass eigen state $\nu_{3}$ is considered to decay invisibly via $\nu_{3} \rightarrow \nu_{s}+J$, where $J$ is a pseudo-scalar and $\nu_{s}$ is a sterile neutrino; and oscillations in the presence of full 3-flavour matter. Since $\nu_{s}$ does not mix with the three active neutrinos, the mixing matrix $U$ in vacuum will be:

$$
U=\left(\begin{array}{ccc}
c_{12} c_{13} & s_{12} c_{13} & s_{13} e^{-i \delta} \\
-c_{23} s_{12}-s_{23} s_{13} c_{12} e^{i \delta} & c_{23} c_{12}-s_{23} s_{13} s_{12} e^{i \delta} & s_{23} c_{13} \\
s_{23} s_{12}-c_{23} s_{13} c_{12} e^{i \delta} & -s_{23} c_{12}-c_{23} s_{13} s_{12} e^{i \delta} & c_{23} c_{13}
\end{array}\right)
$$

where $c_{i j}=\cos \theta_{i j}, s_{i j}=\sin \theta_{i j} ; \theta_{i j}$ are the mixing angles and $\delta$ is the CP violating phase.

Three-flavour evolution equation in the presence of Earth matter, with invisible decay is:

$$
\begin{gathered}
i \frac{d \tilde{\nu}}{d t}=\frac{1}{2 E}\left[U \mathbb{M}^{2} U^{\dagger}+\mathbb{A}_{C C}\right] \tilde{\nu}, \\
\mathbb{M}^{2}=\left(\begin{array}{ccc}
0 & 0 & 0 \\
0 & \Delta m_{21}^{2} & 0 \\
0 & 0 & \Delta m_{31}^{2}-i \alpha_{3}
\end{array}\right), \text { and } \mathbb{A}_{C C}=\left(\begin{array}{ccc}
A_{c c} & 0 & 0 \\
0 & 0 & 0 \\
0 & 0 & 0
\end{array}\right),
\end{gathered}
$$

where $E$ is the neutrino energy, $\alpha_{3}=m_{3} / \tau_{3}$ is the decay constant in units of $\mathrm{eV}^{2}, m_{3}$ is the mass of $\nu_{3}$ and $\tau_{3}$ its rest frame life time. The conversion factor used to make $\alpha_{3}$ to have the units of $\mathrm{eV}^{2}$ is $1 \mathrm{eV} / \mathrm{s}=6.58 \times 10^{-16} \mathrm{eV}^{2}$. Matter potential is

$$
A_{c c}=2 \sqrt{2} G_{F} n_{e} E=7.63 \times 10^{-5} \mathrm{eV}^{2} \rho(\mathrm{gm} / \mathrm{cc}) E(\mathrm{GeV}),
$$

where, $G_{F}$ is the Fermi constant and $n_{e}$ is the electron number density in matter and $\rho$ is the matter density. For anti-neutrinos, the sign of $A_{c c}$ and the phase $\delta$ in Eq. (1.2) will be reversed. 


\subsection{Why use an atmospheric neutrino experiment to probe $\alpha_{3}$ ?}

Since the decay term appears in the oscillation probabilities in the form $\exp (-\alpha L / E)$, no decay corresponds to $\alpha=0$. The effect of $\alpha$ for various values of $L / E$ are shown in Fig. 1; here $\exp (-\alpha L / E)$ vs $L / E$ is plotted for $\alpha=10^{-3}, 10^{-4}, 10^{-5}$ and $10^{-6} \mathrm{eV}^{2}$. The values of $\alpha$ to which a given experiment spanning a specified $L / E$ range is sensitive to, can be understood from here. The red (blue) shaded region in Fig. 1 indicates the $L / E$ range covered by the narrow band NO $\nu$ A neutrino beam with $L=810 \mathrm{~km}$ and $E=1-3 \mathrm{GeV}$ (atmospheric neutrinos at a baseline $L$ $=9700 \mathrm{~km}$ and $E=0.5-25 \mathrm{GeV}$ ). It can be seen from the figure that $\mathrm{NO} \nu \mathrm{A}$ 's sensitivity is limited to larger values of $\alpha$; i.e $10^{-3}$ and $10^{-4} \mathrm{eV}^{2}$ for which the exponential terms shows substantial departure from the no decay value of $\exp (-\alpha L / E)=1$. Since atmospheric neutrino oscillation experiments span an $L / E$ range of $\sim[0.6,25484](\mathrm{km} / \mathrm{GeV})$, they will be sensitive to a wider range of $\alpha$ from $\sim 10^{-6}-10^{-3} \mathrm{eV}^{2}$. Larger $L / E$ values will be sensitive to smaller values of $\alpha$ and vice versa. Since INO ICAL is an atmospheric neutrino detector and has good exposure, it is well suited to probe a phenomenon like invisible neutrino decay. As seen from Fig. 1 ICAL could be sensitive to even $\alpha$ of the order of $10^{-6} \mathrm{eV}^{2}$. The sensitivity to low $\alpha$ values come from low energies, while the higher energies will help in ruling out the larger values of $\alpha$.

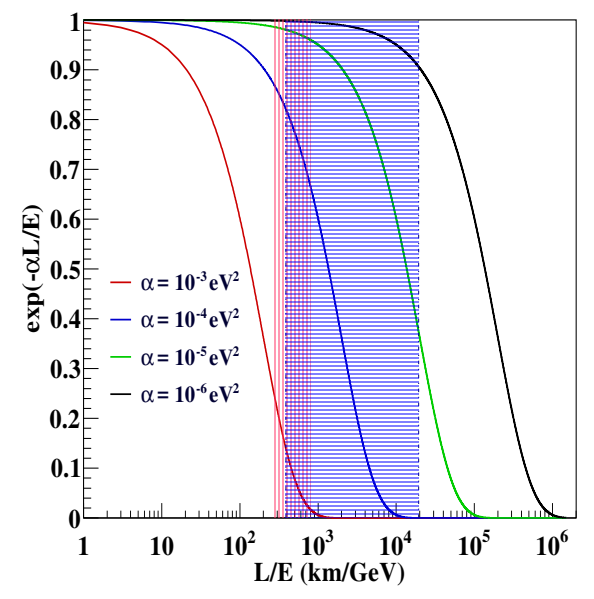

Figure 1: The value of $\exp (-\alpha L / E)$ as a function of $L / E$ for different values of $\alpha$ - from left to right $1 \times 10^{-3}, 1 \times 10^{-4}, 1 \times 10^{-5}$ and $1 \times 10^{-6} \mathrm{eV}^{2}$ respectively. The red shaded region denotes the $L / E$ range accessible with $\mathrm{NO} \nu \mathrm{A}$ narrow band neutrino beam $(E=1-3 \mathrm{GeV})$, the dashed blue shaded region indicates the range for $L=9700 \mathrm{~km}$, when $E$ is in the range $0.5-25 \mathrm{GeV}$.

\section{Three flavour neutrino oscillation probabilities with $\alpha_{3}$}

The three flavour oscillation probabilities in Earth matter, for a baseline of $9700 \mathrm{~km}$ in the presence of invisible decay are shown in Fig. 2. The values of parameters used to generate the oscillation probabilities are : $\delta_{\mathrm{CP}}=0^{\circ} ; \theta_{12}=34.08^{\circ} ; \theta_{23}=39^{\circ}, 45^{\circ}, 51^{\circ} ; \theta_{13}=8.5^{\circ}$; $\Delta m_{21}^{2}=7.6 \times 10^{-5}\left(\mathrm{eV}^{2}\right) ;\left|\Delta m_{32}^{2}\right|=2.4 \times 10^{-3}\left(\mathrm{eV}^{2}\right) ; \alpha_{3}=0,10^{-4}, 10^{-3}\left(\mathrm{eV}^{2}\right)$.

Several things can be understood from the figure. For a given value of $\theta_{23}, \alpha_{3}=0$ corresponds to the no decay case. The effect of decay is to reduce the oscillation probabilities at maxima 

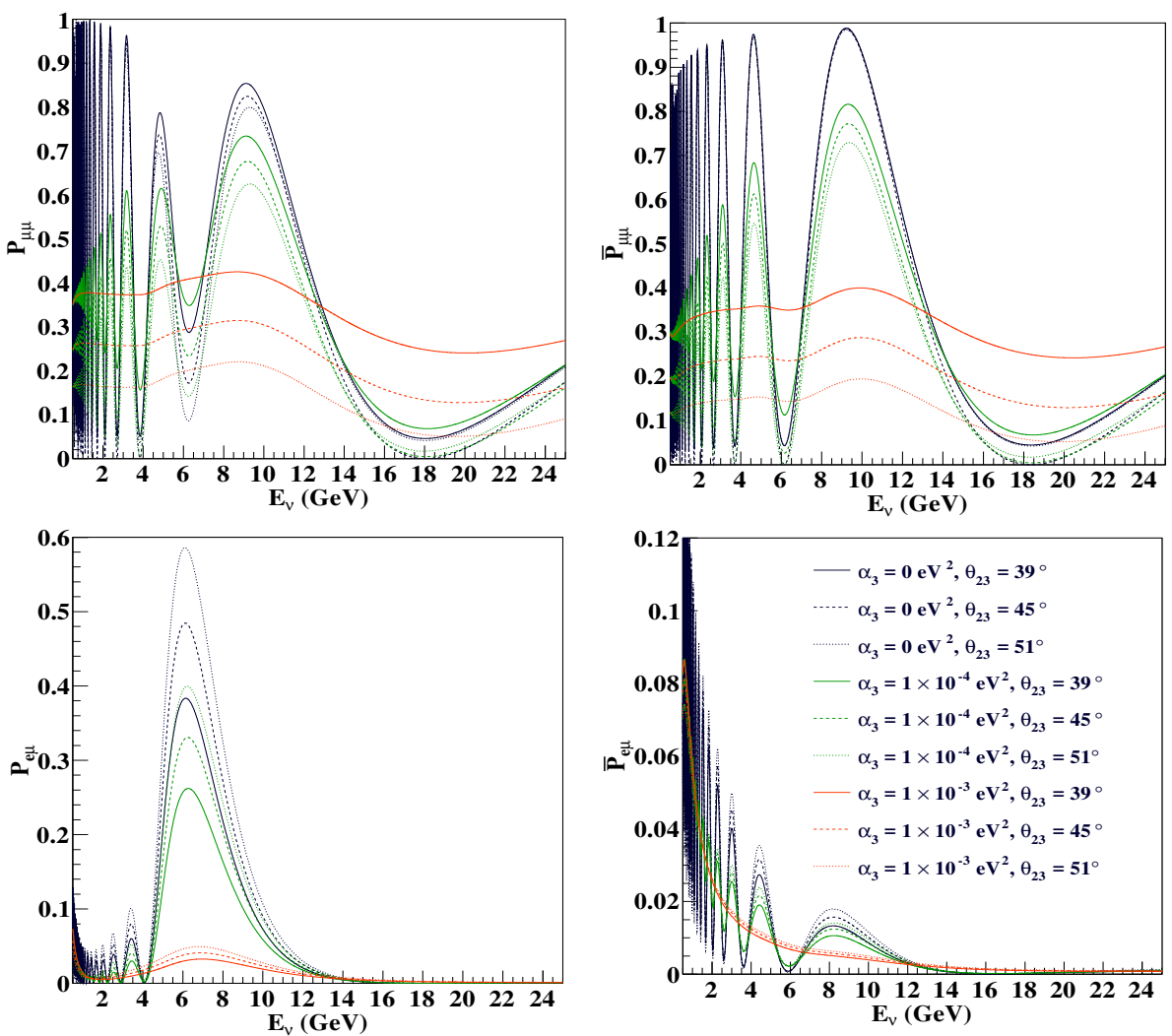

Figure 2: From left to right $P_{\mu \mu}^{m a t t}, \bar{P}_{\mu \mu}^{m a t t}, P_{e \mu}^{m a t t}$ and $P_{\mu \mu}^{m a t t}$ for $L=9700 \mathrm{~km}$ and with different $\theta_{23}$ and $\alpha_{3}$. NH is taken as the true hierarchy.

and elevate at minima, thus causing a damping effect. When $\alpha_{3}$ is very large say $1 \times 10^{-3} \mathrm{eV}^{2}$, oscillations are completely washed out. The larger the value of $\alpha_{3}$, the more the dampening will be. Also the effect is more at lower energies. Since the relative change in $\bar{P}_{\mu \mu}\left(P_{e \mu}\right)$ due to decay is more than that in $P_{\mu \mu}\left(\bar{P}_{e \mu}\right)$, the contribution to the $\alpha_{3}$ sensitivity $\chi^{2}$ will be more from $\bar{\nu}_{\mu}\left(\nu_{\mu}\right)$. But $P_{\mu \mu}$ and $\bar{P}_{\mu \mu}$ being the dominant channels at ICAL, the major contribution to $\alpha_{3}$ sensitivity will come from $\bar{\nu}_{\mu}$ events in the present study.

Since the effect of $\alpha_{3}$ is to alter the amplitude of the oscillations, it will affect the measurement of $\theta_{23}$ which also alters the oscillation amplitudes. This creates a degeneracy between $\alpha_{3}$ and $\theta_{23}$. i.e, $\theta_{23}$ in the first octant combined with a larger (smaller) value of $\alpha_{3}$ can give a probability similar to that with $\theta_{23}$ in second octant + a smaller (larger) value of $\alpha_{3}$ for $P_{\mu \mu}$ and $\bar{P}_{\mu \mu}\left(P_{e \mu}\right.$ and $\bar{P}_{e \mu}$ ). This combined effect affects the sensitivity(discovery potential) to(of) $\alpha_{3}$ and the precision measurement on $\theta_{23}$.

\section{Sensitivity to $\alpha_{3}$}

Sensitivity studies to $\alpha_{3}$ are done with $\nu_{\mu}$ and $\bar{\nu}_{\mu}$ charged current events obtained with 500 kTon years exposure of INO ICAL. The details of the numerical simulations and $\chi^{2}$ analysis can be found in [3,4]. Analyses with fixed parameters as well as marginalisation in the $3 \sigma$ ranges of these parameters were performed. Since there is no real data, the "data" events are simulated with 
a true set of parameters and then fitted with theory events by varying the parameter values. For the sensitivity study of $\alpha_{3}$, "data" was simulated with $\alpha_{3}=0$ and is fitted with theory with different non-zero values of $\alpha_{3}$. The number of oscillated events for different $\alpha_{3}=0,1 \times 10^{-5}, 1 \times 10^{-4}$ $\mathrm{eV}^{2}$, in different bins of $E_{\mu}^{o b s}$ are shown in Fig.3.
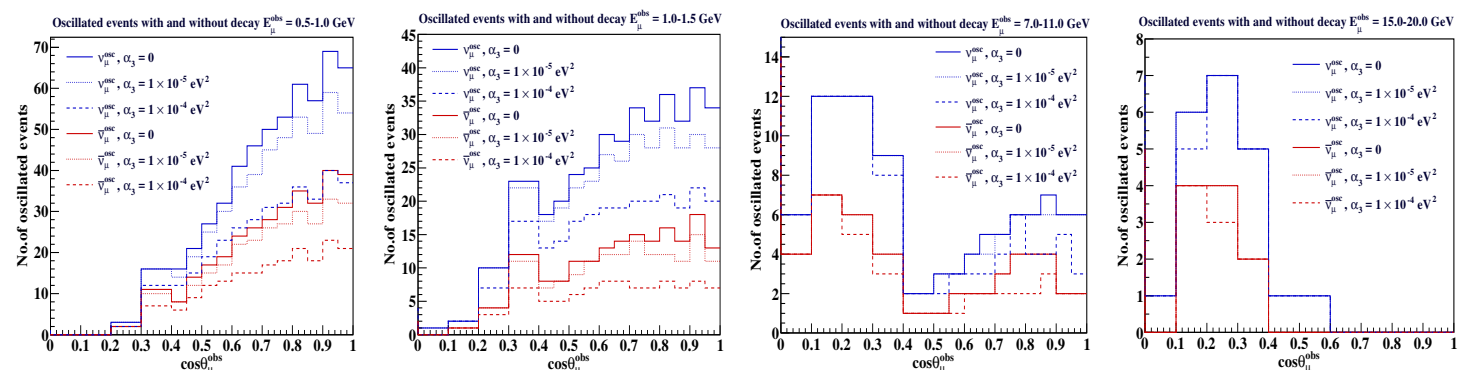

Figure 3: Oscillated $\nu_{\mu}$ and $\bar{\nu}_{\mu}$ events for each $E_{\mu}^{o b s}$ bin as a function of $\cos \theta_{\mu}^{\text {obs }}$ for $\alpha_{3}=0,1 \times 10^{-5}$ and $1 \times 10^{-4} \mathrm{eV}^{2}$. Only up-coming events (oscillated) are shown here.

The sensitivity of 500 kTon year exposure of ICAL with NH (IH) as the true hierarchy is shown in Fig. 4. Previous analyses [5, 6] had obtained a bound of $\alpha_{3}<2.35 \times 10^{-4} \mathrm{eV}^{2}\left(\tau_{3} / m_{3}>\right.$ $2.8 \times 10^{-12} \mathrm{~s} / \mathrm{eV}$ ) at $90 \%$ C.L, with charged current and neutral current events. For true NH, ICAL analysis with charged current $\nu_{\mu}$ and $\bar{\nu}_{\mu}$ events only give bounds of $\alpha_{3}<2.73 \times 10^{-6}$ $\mathrm{eV}^{2}\left(\tau_{3} / m_{3}>2.39 \times 10^{-10} \mathrm{~s} / \mathrm{eV}\right)$ at $90 \% \mathrm{CL}$ with all parameters fixed. With marginalisation over $\theta_{13}, \theta_{23}$ and $\left|\Delta m_{32}^{2}\right|$ the limit at $90 \% \mathrm{CL}$ is $\alpha_{3}<4.36 \times 10^{-6} \mathrm{eV}^{2}\left(\tau_{3} / m_{3}>1.51 \times 10^{-10}\right.$ $\mathrm{s} / \mathrm{eV}$ ) for true NH. This is two orders of magnitude tighter than the bounds given by the previous MINOS and T2K analyses [5, 6]. The results for true IH are similar, with 90\% CL limits of $\alpha_{3}<2.78 \times 10^{-6} \mathrm{eV}^{2}\left(\tau_{3} / m_{3}>2.42 \times 10^{-10} \mathrm{~s} / \mathrm{eV}\right)$ for fixed parameters and $\alpha_{3}<5.82 \times 10^{-6}$ $\mathrm{eV}^{2}\left(\tau_{3} / m_{3}>1.14 \times 10^{-10} \mathrm{~s} / \mathrm{eV}\right)$ with marginalisation.
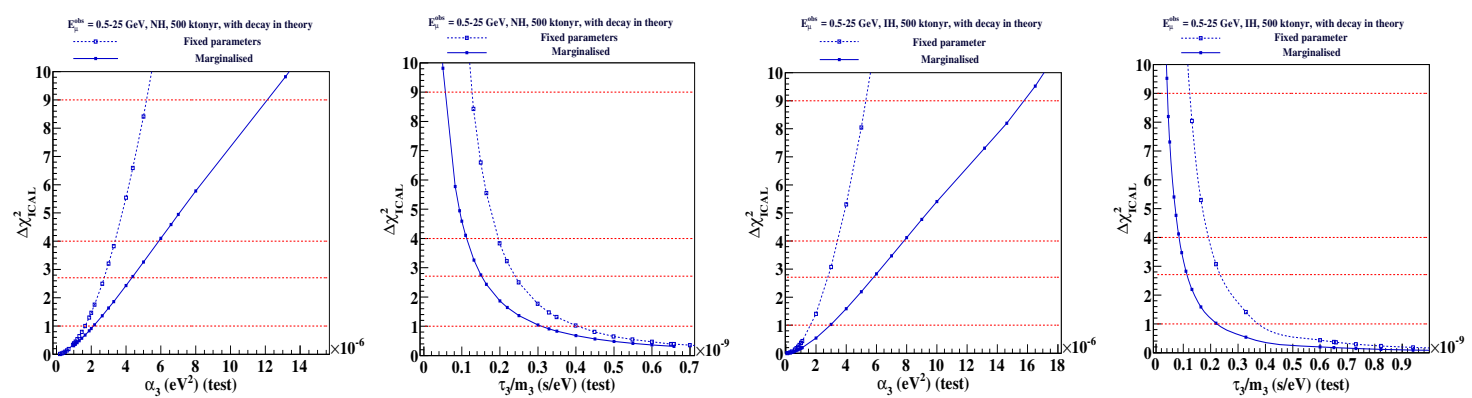

Figure 4: Expected sensitivity of ICAL to neutrino decay with true NH (first and second from the left) and true $\mathrm{IH}$ (third and fourth from the left). The expected $\chi^{2}$ is shown as a function of $\alpha_{3}$ (test) $\mathrm{eV}^{2}$ (panels 1 and 3 ) and $\tau_{3} / m_{3}$ (test) (s/eV) (panels 2 and 4) with 500 kton-yr exposure of ICAL.

\section{Precision measurement of $\sin ^{2} \theta_{23}$ and $\left|\Delta m_{32}^{2}\right|$ in the presence of decay}

The effect of the presence of invisible decay on the precision measurement of $\sin ^{2} \theta_{23}$ and $\left|\Delta m^{2}{ }_{32}\right|$ is also studied. Since decay affects the amplitude of oscillations, it is expected that the 
precision measurement on $\theta_{23}$ will be affected. For these analyses, both "data" and theory are simulated with decay. The value of $\alpha_{3}$ is taken as $1 \times 10^{-5} \mathrm{eV}^{2}$ in "data" and kept at this value in theory for fixed parameter analysis and varied in the range $\left[0-2.35 \times 10^{-4}\right] \mathrm{eV}^{2}$ for marginalised analysis. The comparison with no decay case is also made. The results are shown in Fig. 5.
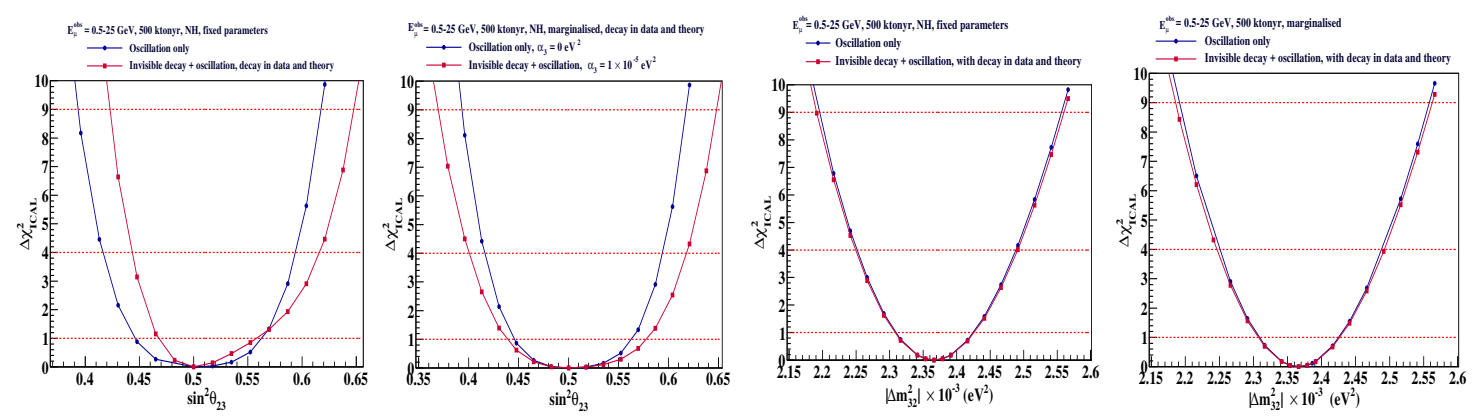

Figure 5: From left to right: Precision on $\sin ^{2} \theta_{23}$ (left-set) and $\left|\Delta m_{32}^{2}\right|$ (right-set) in the presence and absence of invisible decay for fixed parameters and with marginalisation. The value of decay parameter $\alpha_{3}$ in "data" is taken to be $1 \times 10^{-5} \mathrm{eV}^{2}$.

For the fixed parameter case eventhough the relative $1 \sigma$ precision on $\sin ^{2} \theta_{23}$ with $\alpha_{3}=0$ and $1 \times 10^{-5} \mathrm{eV}^{2}$ are similar, $8.9 \%$ for the former and $8.6 \%$ for the latter, the parameter space shifts to the higher $\sin ^{2} \theta_{23}$ side in the with decay case. This can be understood from Fig. 6 .
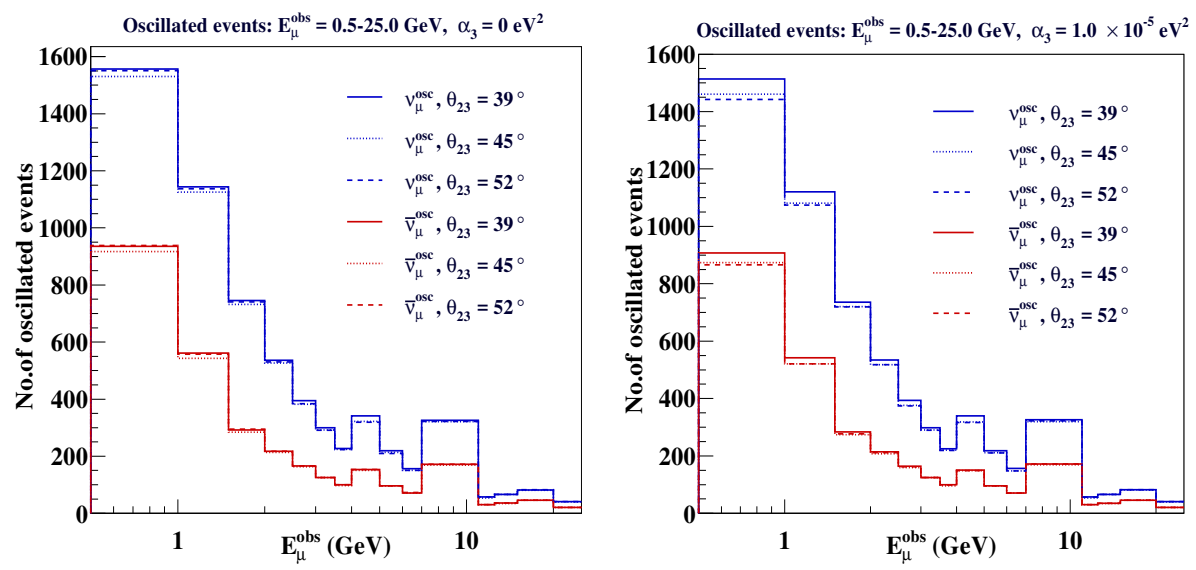

Figure 6: Oscillated $\nu_{\mu}$ events as a function of $E_{\mu}$ for (left) $\alpha_{3}=0 \mathrm{eV}^{2}$ and (right) $\alpha_{3}=1 \times 10^{-5} \mathrm{eV}^{2}$ for $\theta_{23}=39,45$ and $52^{\circ}$.

When there is decay, the difference between the events with $\theta_{23}$ in the first octant and those with $\theta_{23}=45^{\circ}$ is more than that with $\theta_{23}$ in the second octant and those with $\theta_{23}=45^{\circ}$. This causes the parameter space to shift towards the higher $\theta_{23}$ side. When marginalisation is done, the precision without and with decay are $8.9 \%$ and $10.9 \%$ respectively. Here $\alpha_{3}$ can be varied in theory to match the theory events with the "data" generated with true $\theta_{23}=45^{\circ}$.

The precision on $\left|\Delta m_{32}^{2}\right|$ remains the same both in the absence and presence of invisible decay. For fixed parameters the relative $1 \sigma$ on $\left|\Delta m_{32}^{2}\right|$ is $2.5 \%$ whereas with marginalisation it is $2.6 \%$. 


\section{Conclusions}

The sensitivity to invisible neutrino decay with $500 \mathrm{kTon}$ year exposure of ICAL is studied. The analysis with only charged current $\nu_{\mu}$ and $\bar{\nu}_{\mu}$ events give a bound of $\alpha_{3}<2.73 \times 10^{-6} \mathrm{eV}^{2}$ $\left(\alpha_{3}<4.36 \times 10^{-6} \mathrm{eV}^{2}\right)$ at $90 \% \mathrm{CL}$ for fixed parameters (marginalised case), assuming true $\mathrm{NH}$. This is two orders of magnitude tighter than the bounds on $\alpha_{3}$ given by the previous analyses with charged and neutral current events. This means that if we include the analysis of neutral current events also, ICAL should be able to put an even better bound on $\alpha_{3}$. The effect of the presence of decay on the precision of $\sin ^{2} \theta_{23}$ and $\left|\Delta m_{32}^{2}\right|$ was also studied; it was found that the precision on $\sin ^{2} \theta_{23}$ worsens in the presence of invisible decay since it is related to the amplitude of oscillations which the decay also affects.

\section{Acknowledgments}

Prof. Amol Dighe, Prof. D. Indumathi, Prof. S. Uma Sankar and Prof. Jim Libby. LSM acknowledges Nandadevi and Satpura clusters, part of IMSc, Chennai computing facility and thanks the organisers of NUFACT 2017, Anushree Ghosh, Monojit Ghosh and Sushant Raut.

\section{References}

[1] Kumar, A., Vinod Kumar, A.M., Jash, A.et. al., "Invited review: Physics potential of the ICAL detector at the India-based Neutrino Observatory (INO)", Pramana - J Phys 88: 79 (2017).

[2] M. S. Athar et al., "India-based Neutrino Observatory: Project Report. Volume I.", INO-2006-01 (2006).

[3] S. Choubey, S. Goswami, C. Gupta, S. M. Lakshmi and T. Thakore, "Sensitivity to neutrino decay with atmospheric neutrinos at INO,” (2017), arXiv:hep-ph/1709.10376.

[4] Lakshmi. S. Mohan, D. Indumathi, "Pinning down neutrino oscillation parameters in the 2-3 sector with a magnetised atmospheric neutrino detector: a new study", Eur. Phys. J. C 77, 54 (2017), arXiv:hep-ph/1605.04185.

[5] R. A. Gomes, A. L. G. Gomes, O. L. G. Peres, "Constraints on neutrino decay lifetime using long-baseline charged and neutral current data", Physics Letters B 740 (2015).

[6] M. C. Gonzalez-Garcia, Michele Maltoni, "Status of oscillation plus decay of atmospheric and long-baseline neutrinos”, Physics Letters B 663, 405-409 (2008), arXiv:hep-ph/0802.3699. 